Research Article

\title{
Fault Diagnosis of High-Speed Train Bogie Based on Synchrony Group Convolutions
}

\author{
Yunpu Wu $\mathbb{D}$, Weidong Jin, Junxiao Ren, and Zhang Sun \\ School of Electrical Engineering, Southwest Jiaotong University, Chengdu 611756, China \\ Correspondence should be addressed to Yunpu Wu; wu_yunpu@my.swjtu.edu.cn
}

Received 26 July 2019; Accepted 24 September 2019; Published 7 November 2019

Guest Editor: Franco Concli

Copyright (C) 2019 Yunpu Wu et al. This is an open access article distributed under the Creative Commons Attribution License, which permits unrestricted use, distribution, and reproduction in any medium, provided the original work is properly cited.

\begin{abstract}
Health monitoring and fault diagnosis of a high-speed train is an important research area in guaranteeing the safe and long-term operation of the high-speed railway. For a multichannel health monitoring system, a major technical challenge is to extract information from different channels with divergence patterns as a result of distinct types and layout of sensors. To this end, this paper proposes a novel group convolutional network based on synchrony information. The proposed method is able to gather signals with similar patterns and process these channels with specific groups of neurons while simultaneously assigning signals with significant difference to different groups. In this approach, the feature can be extracted more effectively and the performance can be improved, owing to the sharing of filters for similar patterns. The effectiveness of the method is validated on high-speed train fault dataset. Experiments show that the proposed model performs better than normal convolutions and normal group convolutions on this task, which achieves an accuracy of $98.27 \%(\sigma=1.73)$ with good computational efficiency.
\end{abstract}

\section{Introduction}

As a rapid developing modern transportation method in the world, the high-speed train has the advantages of fast, efficient, and environment friendly. With the high speed, high density, and cross-regional development of high-speed trains, the safe operation and maintenance of high-speed trains has become the first concern of the field [1-3]. During long-term operation of high-speed trains, the performance deterioration of critical components would threaten the safety of the vehicle. The high-speed train bogie is the only component that connects the vehicle body and the track (as shown in Figure 1). In the suspension system of the bogie, there would be deteriorating of rubber nodes, oil leakage of the dampers, air leakage of air spring, and breakage of the axle box spring, which would endanger the safety of operation.

The condition monitoring and fault diagnosis for critical components are an urgent need for high-speed trains. However, the number of sensor channels on the train body for vibration monitoring signals could be considerable. There are also couplings between sensor signals due to wheel-rail contact force, friction, and interaction between suspension systems $[4,5]$. Besides, the vibration of highspeed trains is also affected by many influencing factors, including environment and load [6-9]. Therefore, highspeed train monitoring signal analysis not only needs to deal with high dimensions and high complexity but also many uncertain factors. Some efforts have been performed for fault diagnosis of high-speed train, including suspension system $[10-13]$, traction system $[14,15]$, wheelset bearing system $[16,17]$, and on-board equipment $[18,19]$. Gasparetto et al. [20] proposed a data-driven approach for detecting the incipient instability of the bogie, which is based on random decrement technique and Prony method. Chen et al. [21] proposed a probability-relevant PCA method for detecting incipient sensor faults in electrical drive systems of highspeed trains. Zhuang et al. [22] developed a combined method based on convolution sparse representation, Hilbert transform, and manifold learning for detecting bearing faults of trains.

Deep neural network, as an efficient machine learning model, has achieved great success in many fields such as computer vision, natural language processing, and 


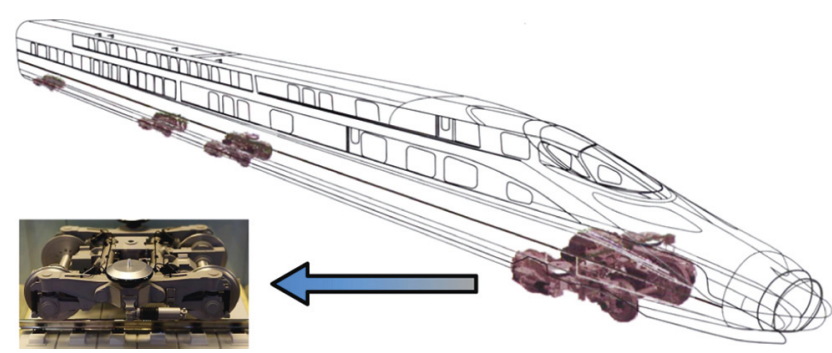

Figure 1: The bogie units of the high-speed train.

automatic driving $[23,24]$. For signal analysis and recognition, the deep learning architecture can not only deal with high-dimensional data but also reduce the dependence on expert experience and domain knowledge [25, 26]. Some scholars have employed deep neural networks in condition monitoring of high-speed trains [27, 28]. Hu et al. [29] used an autoencoder to obtain fault features of the bogie and then combined deep neural network faults for classification. Peng et al. [30] employed one-dimensional convolutional neural networks to identify wheelset faults in high-speed trains. In [31], a multiperspective neural network was proposed for the multichannel and multicomponent signal analysis, which extract features from perspectives of channel, component, and timescale. However, the monitoring sensors on the vehicle are different not only in measure but also in different positions [32], which makes the pattern of the monitoring signals have group disparities. For conventional deep neural networks, it is tricky to make manual adjustments for input channels. Therefore, the divergences and convergences among signals in different channels present a challenge to the fault diagnosis model based on deep neural networks.

Correlation analysis (also known as synchrony analysis in the signal process) is a method to evaluate the relationship between two or more variables. It can be used to explore potential similarities of signal patterns. There have been some efforts to apply correlation analysis in monitoring signal analysis. Liu et al. [33] employed complex continuous wavelet coherence to quantify the intense correlation of motor current and chatter vibration, which require a wide bandwidth of frequency response. Hong and Huang [34] evaluated the health condition of the power transformer through the vibration correlation analysis. Kestner et al. [35] analysed the correlations for sensors between the pressure measurements and bearing vibration measurements of gas turbine compressor from the view of harmonics. Recent studies suggested that the analytic representation can be another way to obtain time-resolved connectivity of signals. Córdova-Palomera et al. [36] employed analytic components of the signal to quantify the oscillatory synchronization of cerebellar functional networks. Pedersen et al. [37] applied instantaneous phase synchrony analysis to obtain phase information between all possible node-wise time series and compared its performance with correlation-based sliding window analysis. Hence, analytic representation of signals can capture the temporal resolution synchrony among signals, which provide a feasible approach for similarity measure of monitoring signals.
This paper proposes a fault diagnosis structure for the multichannel monitoring system to address the issues brought by the divergences and convergences of different sensors in signal patterns. We combine group convolutions with synchrony information to improve the ability to process multichannel signals with group disparities. The proposed method is able to collect signals with similar patterns and process them with specific groups of neurons, which could extract features more effectively. On the contrary, signals with significant differences would be assigned to different groups of neurons, instead of sharing the same neurons, which makes the model much easier to optimize. The contributions of this paper are as follows:

(1) Three synchrony measurements (instantaneous phase synchrony, amplitude envelope synchrony, and composite synchrony) are introduced to estimate the similarity between vibration signals, which provide a scalable and flexible approach to measure the synchronization of multichannel signals.

(2) A synchrony group convolutional network is proposed for the signal pattern analysis and feature extraction of the multichannel monitoring system. The proposed structure can process multichannel signals with strong coupling and complex group disparities.

(3) The proposed method is applied to fault diagnosis of a high-speed train bogie. The results show that our scheme can achieve high accuracy of fault classification and reduce the model size and burden of computation, which provides a feasible and practical structure for fault diagnosis of the multichannel monitoring system.

The remainder of this paper is organized as follows: Section 2 has three distinct subsections: Sections 2.1, 2.2, and 2.3 give brief introductions to synchrony quantification, hierarchical clustering, and group convolutions in neural networks. Section 3 describes the proposed synchrony group convolutional network for multisensor-based fault diagnosis. Section 4 presents the experimental results for bogie fault diagnosis of the high-speed train. Finally, conclusions and some perspectives are presented in Section 5.

\section{Methods}

In this section, the principles of synchrony analysis and hierarchical clustering for similarity-based grouping of sensor channels are presented. Furthermore, group convolutions are introduced, which is a variant of convolution layers in neural networks.

\subsection{Synchrony Analysis and Correlation Quantification.} Signal synchrony $[37,38]$ can reveal the independence, conditioning, and correlations between two or sometimes more signals, and it can be characterized in many different ways. In this section, two correlation metrics for synchrony analysis between signals are presented: the amplitude envelope and the instantaneous phase (corresponding to $|a(t)|$ 
and $\varphi(t))$, which both are derived from analytic representation [39].

Let $Y$ be a two-dimensional matrix of size $N \times T$ including signals across $N$ channels where $T$ is the length of the signal. $z_{1}[t]$ and $z_{2}[t]$ are the analytic representations of rows in $Y$, i.e., $y_{1}[t]$ and $y_{2}[t]$, respectively; that is,

$$
\begin{aligned}
& z_{1}[t]=y_{1}[t]+j \tilde{y}_{1}[t]=a_{1}[t] e^{j \varphi_{1}[t]}, \\
& z_{2}[t]=y_{2}[t]+j y_{2}[t]=a_{2}[t] e^{j \varphi_{2}[t]},
\end{aligned}
$$

where $j=\sqrt{-1}, \tilde{y}$ denotes the Hilbert transform of $y, a_{1}[t]$ and $a_{1}[t]$ denote the instantaneous amplitudes, $\varphi_{1}[t]$ and $\varphi_{2}[t]$ are the instantaneous phases of $y_{1}[t]$ and $y_{2}[t]$, respectively,. The instantaneous phase $\varphi[t]$ and amplitude envelope $a[t]$ comprise distinct correction information among channels, respectively. The instantaneous phase contains the temporal oscillating properties of signals, while the amplitude envelope can represent the varying level of signals over time.

In order to capture the synchrony between signals, the Pearson correlation coefficient [40] is introduced to qualify the amplitude envelope synchrony between signals, which is a statistical measure of the strength of a linear relationship between paired data and can indicate the covarying of two continuous signals. The Pearson correlation coefficient between the amplitude envelopes can be calculated as follows:

$$
r=\frac{\sum\left(a_{1}[t]-m_{1}\right)\left(a_{2}[t]-m_{2}\right)}{\sqrt{\sum\left(a_{1}[t]-m_{2}\right)^{2} \sum\left(a_{2}[t]-m_{2}\right)^{2}}},
$$

where $m_{1}$ is the mean of vector $a_{1}[t]$ and $m_{2}$ is the mean of the vector $a_{2}[t]$. The values of the Pearson correlation coefficient range from -1 to 1 . The positive value denotes two signals have positive correlation and vice versa. An additional consideration is that we are more concerned with the strength of the correlation between signals for the purpose. Hence, the absolute value of the Pearson correlation coefficient, which ignores the sign, is employed as an indicator of the relationship between signals.

The Pearson correlation coefficient is a global synchrony measurement that reduces the relationship between two signals to a single value. However, it is equally essential to analyse the local synchrony in synchrony analysis. One way to obtain the time-resolved Pearson correlation is to calculate the coefficient with the sliding window, which partitions the time-domain input signal into several disjointed or overlapped blocks by multiplying the signal with a window function until the entire signal is covered. For local synchrony of the instantaneous phase, there is another measurement for signal synchrony. Instantaneous phase synchrony analysis is a method for deriving time-resolved connectivity analysis of signals, which has been applied in functional connectivity analysis [37] and could characterize the phase synchrony at the same temporal resolution as the input signal.

Assuming that signals satisfy Bedrosian's theorem [41], the two signals $y_{1}[t]$ and $y_{2}[t]$ are phase locked of order $1: 1$ when

$$
\left|\varphi_{1}[t]-\varphi_{2}[t]\right| \approx 0,
$$

where $|\cdot|$ is the absolute value operator. The instantaneous phase difference between the signal pairs $\varphi_{1}[t]$ and $\varphi_{2}[t]$, which obtained from all pairs of rows in the analytic associate matrix, can be calculated as follows:

$$
i P C_{1,2}[t]=\operatorname{abs}\left(\sin \left(\varphi_{1}[t]-\varphi_{2}[t]\right)\right) .
$$

The sinusoid accounts for phase wrapping and ambiguity in the sign of phases over time. Calculation of equation (4) for all pairs of channels and all-time points yields a three-dimensional matrix $D=\left[i P C_{1,2}[t]\right] \in \mathbb{R}^{N \times N \times T}$. At each time point, the phase coherence quantity $(1-i P C)$ between signal pairs is calculated, ranging from 0 (no phase coherence) to 1 (maximal phase coherence).

In this paper, the global level synchrony of the phase synchrony and the amplitude envelope between each combination is quantified by the Pearson correlation coefficient and average phase coherence for all time steps, respectively. Besides, the composite synchrony among channels is calculated through the weighted average of amplitude envelope synchrony and instantaneous phase synchrony. This average approach and its relationship with hierarchical clustering are explained in the following section.

2.2. Hierarchical Clustering. The hierarchical clustering [42] is a hierarchical decomposition method based on group similarities. Hierarchical clustering builds a cluster hierarchy, which is widely known as the dendrogram (Figure 2), representing the nested grouping of patterns and similarity levels at which groupings change. The clustering process is performed by merging the most similar patterns in the cluster set to form a bigger one. The agglomerative hierarchical clustering which is the most common hierarchical clustering based on the bottom-up approach is employed in this paper. It considers each data point as an individual cluster. At each iteration, the similar clusters merge with other clusters until one cluster is formed.

The basic scheme of the agglomerative hierarchical clustering is shown as follows:

(1) Clusters initialization: each data point is considered as an individual cluster

(2) Clusters distance (similarity) calculation: all pairwise distances are calculated according to the similarity, which results in a distance matrix

(3) Combine the foremost closed two clusters

(4) Update the distance matrix to replicate the pairwise distance between the new cluster and the original clusters

(5) Repeat Steps 3 and 4 until only a single cluster remains

Agglomerative hierarchical clustering relies on constructing a distance matrix between all of the clusters. A critical operation is to determine the distance of two clusters. There are various approaches which are used to calculate the distance between two clusters. The most common ways include single linkage, complete linkage, and average linkage [43]. Single linkage hierarchical clustering defines the shortest 


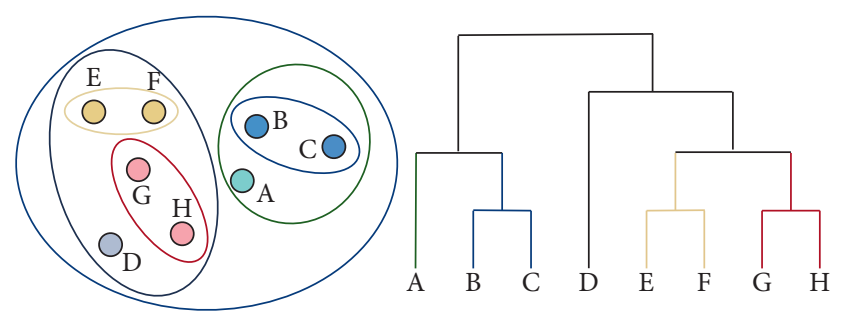

FIGURE 2: Hierarchical clustering.

distance between two points in each cluster as the distance between two clusters. Complete linkage hierarchical clustering defines the longest distance between two points in each cluster as the distance between two clusters, while average linkage is a compromise solution to measuring the distances of clusters. The distance between two clusters is the average of the pairwise distance between points in the two clusters. The cluster distance between clusters $\mathrm{A}$ and $\mathrm{B}$ is defined as

$$
D_{A B}=\frac{1}{|A||B|} \sum_{a \in A} \sum_{b \in B} d[a, b],
$$

where $d[\cdot]$ represents the distance measurement between points. The composite synchrony, which has been mentioned in Section 2.1, is the weighted average of amplitude envelope synchrony and instantaneous phase synchrony. This approach is equivalent to the average of two distance measurement between clusters, and thus it can be directly taken as the distance measurement for hierarchical clustering.

There are two main advantages of hierarchical clustering for our purpose. Firstly, it makes it possible to obtain clusters by precomputed pairwise distance matrix, which is compatible with the measure of pairwise synchrony and ease of handling of any types of similarity or distance. Secondly, the hierarchical clustering can embed flexibility concerning the extent of granularity. A sensor channel may belong to different clusters under different viewpoints. For example, vibration sensors on the vehicle can be divided into displacement sensors and accelerometers based on measured values. Alternatively, these sensors can also be grouped by the measured directions, namely, lateral direction, vertical direction, and longitudinal direction. The desirable hierarchy and number of clusters could be obtained by "cutting" the dendrogram at the proper level, which makes it convenient to adjust the number of clusters according to requirements. Thus, the agglomerative hierarchical clustering can be applied to obtain the groups of sensor channels from signal synchronicity.

2.3. Group Convolutions. Group convolutions are a variant of convolution layer in neural networks where the channels of the input feature map are grouped, and the convolution operation is performed independently for each channel group. Usually, convolution filters are applied on an input layer by layer to get the final output feature maps. Instead of applying all filters on all channels, group convolutions use different sets of convolution filter groups on the same input so that there is more than one pathway for convolutions on a single input (Figure 3). The initial use of this idea of group

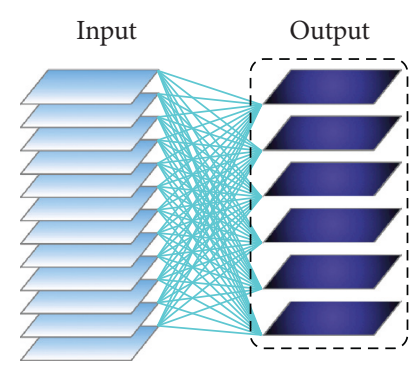

Convolution

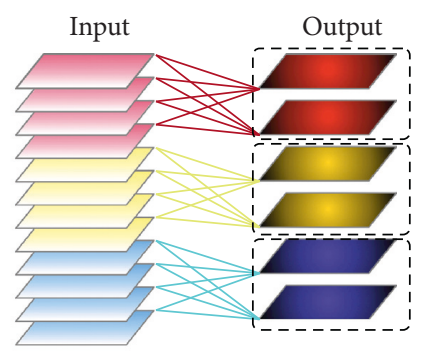

Group convolution
Figure 3: Normal convolution and group convolution.

convolutions can be noticed in the study of Krizhevsky et al. [44], through which deep neural networks can be trained with less resource requirement for processor and memory.

The main advantages of group convolution are as follows [45]:

(1) Reducing model size: The group convolutions can decrease the model parameters by filter grouping. Moreover, for a convolution layer with $c_{1}$ input channels and $c_{2}$ output channels, the group convolution with $g$ independent groups can reduce filter dimensions from $h \times w \times c_{1}$ to $h \times w \times c_{1} / g$, in which $c_{2} / g$ filters operate on $c_{1} / g$ channels. Through that, the computational complexity and the number of model parameters can be significantly reduced.

(2) Efficient training process: By the group approach, the convolution operations are divided into multiple independent parts and can be handled by different processors in a parallel way. The parallel execution can reduce the training time and improve scalability of the model.

(3) Better feature representation: Group convolutions can also provide a better model than normal convolutions. Through the filter group structure, feature maps are forced into a dense block diagonal structure, and filters with strong mutual information are grouped adjacent to each other.

In this paper, instead of dividing channels into equal groups sequentially, the groups of sensor channels are inferred based on the signal correlation from amplitude envelope synchrony and phase synchrony.

\section{Proposed Fault Diagnosis Framework Based on Synchrony Group Convolutions}

This paper focuses on improving the ability of the neural network in processing multichannel signals with group disparities. Because of different settings of monitoring sensors in types and locations, there would be divergences and convergences among signals in different channels. The diversity of channel signals would grow with increase in the channel number, which requires more effective neural network model for feature extraction.

In order to address this issue, we propose synchrony group convolutions to construct a fault diagnosis scheme for 
a high-speed train bogie. We combine group convolutions with synchrony information to improve the ability to process multichannel signals with group disparities. A complete description of the proposed structure for fault diagnosis is schematically represented in Figure 4. Different from conventional group convolutions, the proposed method does not require that each group has the same number of filters because the channels are grouped based on synchrony information. The structure details and process are as follows:

(1) Synchrony calculation: the pairwise phase synchrony and amplitude envelope synchrony between sensor channels are calculated based on analytic components and correlation measurement. The composite synchrony between signals is obtained through a weighted average of these two correlation metrics derived from the Pearson correlation coefficient and the average phase coherence, respectively.

(2) Channel clustering based on synchrony: after obtaining the synchrony information between channels, we use the pairwise correlations to construct a correlation matrix. Then, agglomerative hierarchical clustering is employed to cluster channels with strong correlations. It should be noted that the group number should be adjusted taking into account not only clustering results but the complexity of the neural network.

(3) Synchrony group convolutional network training: in the fault diagnosis model, channel groups in group convolution layers are based on the cluster results of hierarchical clustering. Synchrony group convolutions are configured in the forepart of the network. The filters in each group keep independent in these layers. At a more specific level, the output feature maps for a certain group would still be in one group in the next convolution layer, which could extract much deeper features for a channel group with synchrony signals. After all synchrony group convolution layers, standard convolution layers are attached for feature fusion.

The proposed structure is an entire end-to-end neural network that all synchrony group convolution layers can be freely adjusted. It should be added that the proposed synchrony group convolutions are compatible with existing deep structures of neural networks. Synchrony group convolution layers can be plugged into the forepart of established structures, which can improve the capabilities of the neural network model for handling multisensor signals. The main advantages of the proposed scheme are concluded as follows:

(1) Correlated pattern assembly: The synchrony group convolutions can discover and extract the latent features for signals with similar patterns, which improve the performance and capacity of the neural network for multichannel signals.

(2) Scalable and adjustable: The synchrony group convolution layers can be configured on the basis of actual requirements of signal channels. The depth and width of these layers are fully controllable. The group's number can also be adjusted through proper hierarchy choice.

(3) Fully learnable structure: The entirely data-driven scheme reduces the requirement for prior knowledge and needs few human interventions, which also reduces the biases caused by limited domain knowledge.

\section{Experimental Results and Analysis}

4.1. Data Description. The data for experiments is from the simulation platform of the high-speed train bogie, which is developed by the State Key Laboratory of Traction Power at Southwest Jiaotong University [31]. The simulation platform is based on the nonlinear dynamic model of an electric multiple-unit system with the multibody dynamics analysis software Simpack. The geometric nonlinearity of wheel-rail contact, the creep nonlinear, and the nonlinear suspension of wheel-rail are all taken into account. The track irregularity spectrum, obtained by measuring the high-speed railway in China, is imposed as inputs and excitations of the simulation model. Suspension parameters of the bogie in the simulation model are consistent with the high-speed train CRH380A. Hence, the monitoring signals under this track spectrum can be viewed as a maximum approximation to real environments, with track irregularity, and other factors contained.

The monitoring sensors measure the motion characteristics, including lateral, longitudinal, and vertical vibration accelerations of different parts (Figure 5) of the vehicle and displacements of the body, the bogie, and the wheelset. Table 1 gives all descriptions to the 58 channels of the dataset. The data are collected at a sampling frequency of 243 samples per second from 58 channels. Five categories of fault are considered in the experiments, which are air spring fault, wheel-box spring fault, lateral damper fault, yaw damper fault, and vertical damper fault. Considering there are multiple same type components in the locomotive frame (e.g., there are four yaw dampers for each bogie), there would be 30 types of fault in classification.

4.2. Channel Synchrony and Hierarchical Clustering. In order to explore the correlation between channels, the pairwise synchrony for all combinations of 58 sensor channels is calculated, both the instantaneous phase and the amplitude envelope. Here, two examples of the channel synchrony calculation are presented, which are local synchrony and global synchrony in the instantaneous phase and the amplitude envelope. The results of synchrony analysis are illustrated in Figure 6.

The first example is channel 11 and channel 20 which is shown in Figure 6(a). It can be seen from Table 1 that two sensor channels measure the lateral acceleration, respectively, in the middle of bogie 1 and axle box 1 . From the results, it can be seen that these two channels have significant synchrony in both instantaneous phase (0.65) and amplitude envelope (0.48), as a result of the close spatial location and the same measurement direction. However, the position 


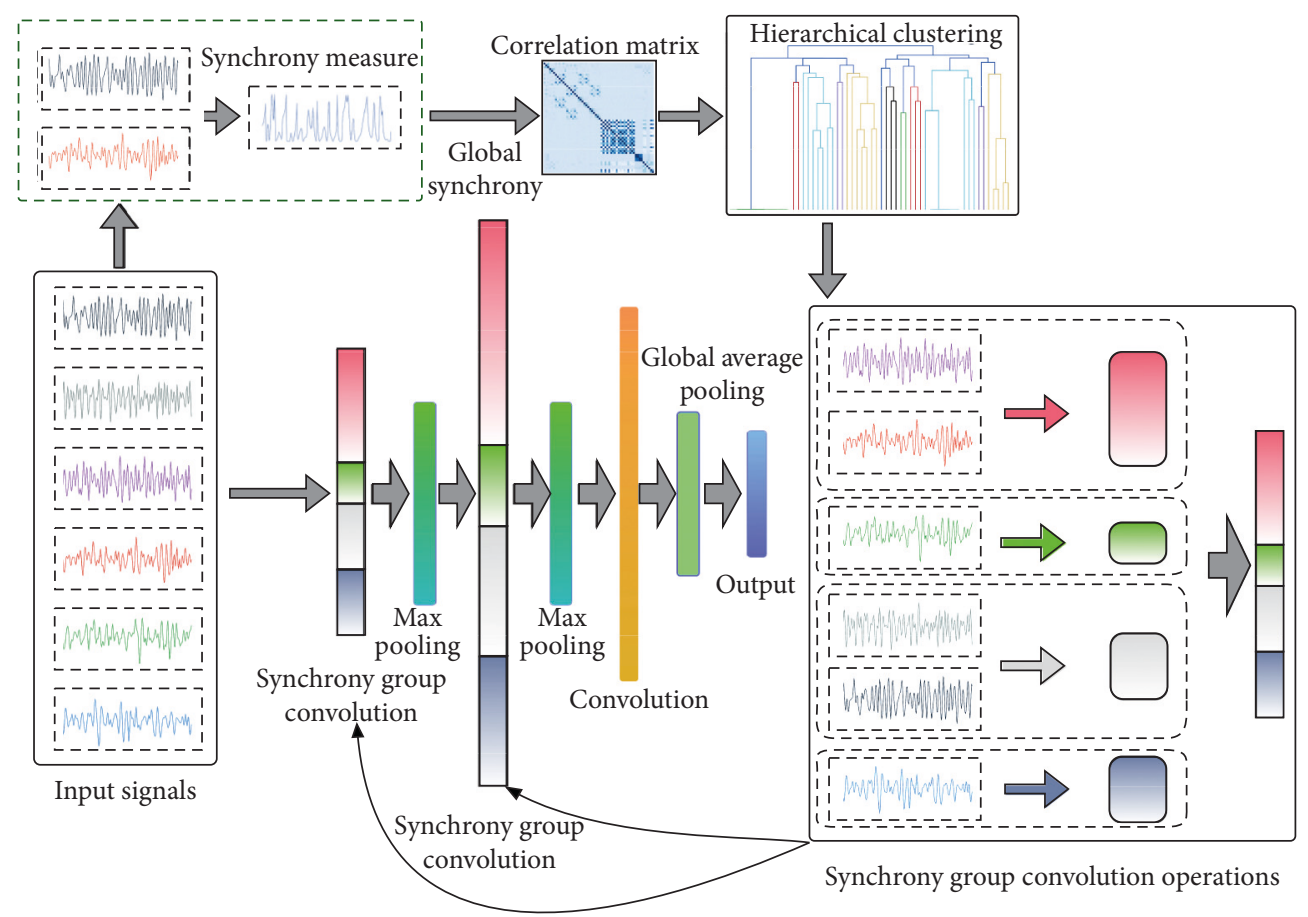

FIgURE 4: The proposed framework based on synchrony group convolutions for fault diagnosis of the high-speed train.

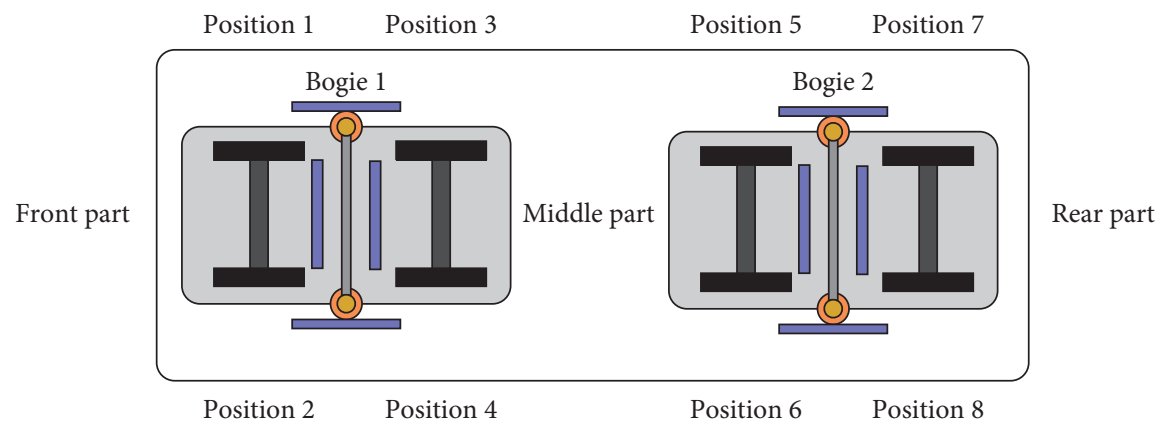

Figure 5: The structure of the high-speed train bogie and the location of sensors.

difference leads to the amplitude difference, which lowers the amplitude envelope synchrony. Another example is the lateral acceleration (channel 11) and the vertical acceleration (channel 12) of the bogie 1 in the middle which is shown in Figure 6(b). We can see that even the position of two sensor channels is completely identical and the synchrony between them is weak with respect to the orthogonality of measurement direction. The results of the synchrony analysis for these two examples are consistent with our domain knowledge.

Through calculating phase synchrony and amplitude envelope synchrony of all combination of 58 sensor channels, the correlation matrix is available. According to the correlation matrix, clusters of sensor channels can be obtained through the hierarchical clustering, which is shown in Figure 7. It can be seen from the cluster results that hierarchical clustering can capture the group's correlations among multichannel signals. As mentioned previously, hierarchical clustering can obtain the desired number of clusters in engineering applications. Phase synchrony and amplitude envelope synchrony tend to gather channels with common characteristics from their individual perspectives. In comparisons, the composite synchrony provides an integrative perspective for synchrony between channels through the weighted average of two measurements, which can be regarded as an information synthesis in distance measurement for hierarchical clustering.

4.3. Fault Diagnosis for High-Speed Train Bogie. Our experiments compare the proposed synchrony group convolutions with the normal convolution and normal group convolutions (GC) to validate the performance of the proposed scheme. We also compare the performance of synchrony group convolutions based on three measurements, namely, amplitude envelope synchrony (ASGC), instantaneous phase synchrony (PSGC), and composite synchrony (CSGC). 
TABle 1: Channel descriptions of monitoring sensors on the vehicle.

\begin{tabular}{|c|c|}
\hline $\begin{array}{l}\text { Channel } \\
\text { number }\end{array}$ & Description \\
\hline 1 & Lateral acceleration of the vehicle front part \\
\hline 2 & Lateral acceleration of the vehicle rear part \\
\hline 3 & Lateral acceleration of the vehicle middle part \\
\hline 4 & Vertical acceleration of the vehicle middle part \\
\hline 5 & Vertical acceleration of the vehicle front part \\
\hline 6 & Vertical acceleration of the vehicle rear part \\
\hline 7 & Lateral acceleration of the bogie 1 in position 1 \\
\hline 8 & Vertical acceleration of the bogie 1 in position 1 \\
\hline 9 & Lateral acceleration of the bogie 1 in position 4 \\
\hline 10 & Vertical acceleration of the bogie 1 in position 4 \\
\hline 11 & Lateral acceleration of the bogie 1 in the middle \\
\hline 12 & Vertical acceleration of the bogie 1 in the middle \\
\hline 13 & Lateral acceleration of the bogie 2 in position 5 \\
\hline 14 & Vertical acceleration of the bogie 2 in position 5 \\
\hline 15 & Lateral acceleration of the bogie 2 in position 8 \\
\hline 16 & Vertical acceleration of the bogie 2 in position 8 \\
\hline 17 & Lateral acceleration of the bogie 2 in the middle \\
\hline 18 & Vertical acceleration of the bogie 2 in the middle \\
\hline 19 & Longitudinal acceleration of the axle box 1 \\
\hline 20 & Lateral acceleration of the axle box 1 \\
\hline 21 & Vertical acceleration of the axle box 1 \\
\hline 22 & Longitudinal acceleration of the axle box 2 \\
\hline 23 & Lateral acceleration of the axle box 2 \\
\hline 24 & Vertical acceleration of the axle box 2 \\
\hline 25 & Longitudinal acceleration of the axle box 3 \\
\hline 26 & Lateral acceleration of the axle box 3 \\
\hline 27 & Vertical acceleration of the axle box 3 \\
\hline 28 & Longitudinal acceleration of the axle box 4 \\
\hline 29 & Lateral acceleration of the axle box 4 \\
\hline 30 & Vertical acceleration of the axle box 4 \\
\hline 31 & $\begin{array}{l}\text { Longitudinal displacement of the vehicle front } \\
\text { part }\end{array}$ \\
\hline 32 & Vertical displacement of the vehicle front part \\
\hline 33 & $\begin{array}{l}\text { Longitudinal displacement of the vehicle middle } \\
\text { part }\end{array}$ \\
\hline 34 & Vertical displacement of the vehicle middle part \\
\hline 35 & $\begin{array}{c}\text { Longitudinal displacement of the vehicle rear } \\
\text { part }\end{array}$ \\
\hline 36 & Vertical displacement of the vehicle rear part \\
\hline 37 & Lateral displacement of the bogie 1 in position 1 \\
\hline 38 & Vertical displacement of the bogie 1 in position 1 \\
\hline 39 & Lateral displacement of the bogie 1 in position 4 \\
\hline 40 & Vertical displacement of the bogie 1 in position 4 \\
\hline 41 & Lateral displacement of the bogie 1 in the middle \\
\hline 42 & $\begin{array}{l}\text { Vertical displacement of the bogie } 1 \text { in the } \\
\text { middle }\end{array}$ \\
\hline 43 & Lateral displacement of the bogie 2 in position 5 \\
\hline 44 & Vertical displacement of the bogie 2 in position 5 \\
\hline 45 & Lateral displacement of the bogie 2 in position 8 \\
\hline 46 & Vertical displacement of the bogie 2 in position 8 \\
\hline 47 & Lateral displacement of the bogie 2 in the middle \\
\hline 48 & $\begin{array}{l}\text { Vertical displacement of the bogie } 2 \text { in the } \\
\text { middle }\end{array}$ \\
\hline 49 & Lateral displacement of the wheelset 1 \\
\hline 50 & Lateral displacement of the wheelset 2 \\
\hline 51 & Lateral displacement of the wheelset 3 \\
\hline 52 & Lateral displacement of the wheelset 4 \\
\hline 53 & $\begin{array}{l}\text { Relative displacement of the primary suspension } \\
\text { in position } 1\end{array}$ \\
\hline
\end{tabular}

TABle 1: Continued.

\begin{tabular}{|c|c|}
\hline $\begin{array}{l}\text { Channel } \\
\text { number }\end{array}$ & Description \\
\hline 54 & $\begin{array}{l}\text { Relative displacement of the primary suspension } \\
\text { in position } 8\end{array}$ \\
\hline 55 & $\begin{array}{c}\text { Relative displacement of the secondary } \\
\text { suspension in position } 1\end{array}$ \\
\hline 56 & $\begin{array}{l}\text { Relative displacement of the secondary } \\
\text { suspension in position } 4\end{array}$ \\
\hline 57 & $\begin{array}{l}\text { Relative displacement of the yaw damper in } \\
\text { position } 1\end{array}$ \\
\hline 58 & $\begin{array}{l}\text { Relative displacement of the yaw damper in } \\
\text { position } 8\end{array}$ \\
\hline
\end{tabular}

As mentioned before, the monitoring data come from multibody dynamics simulations under activation of the track spectrum. The data samples for experiments are processed by the sliding window with 243 points width. For data in the training set, the window is slid by 20 points. For data in the valid set and the test set, the window is slid by 243 points, which is equal to window length and no overlapping. There are a total of 17980 samples which contain 3100 training samples, 7440 validation, and 7440 testing samples, and each class at each speed has an equal number of samples.

In addition, for proposed synchrony group convolutions and normal group convolutions, we compare the performance under 4 groups, 8 groups, and 12 groups, respectively, to test the model performance under various conditions. For the sake of comparison, only synchrony group convolution layers are replaced with normal convolution layers in corresponding comparative experiments, the structure and parameters being maintained. We conduct ten trials with random split training sets to obtain the average accuracy and standard deviation. The experiment results are reported in Table 2. The training curves with error bands for experiments are also presented in Figure 8.

It can be seen from the results in Table 2 that synchrony group convolutions show superior performance and significantly improve the performance when dealing with multichannel signals compared with normal convolution and normal group convolutions, especially the experiment groups based on amplitude envelope synchrony and composite synchrony. The only exception is the experiment 8 , in which channels are divided into 4 groups based on instantaneous phase synchrony. It implies that the instantaneous phase synchrony method may require finegrained groups. As the experimental data display, compared with convolutions without groups, normal group convolutions could improve the accuracy (experiment 2) and may decrease the performance (experiments 3 and 4) of the model under improper group numbers.

It is worth to mention that each synchrony measurement has its own merits (experiments 7, 9, and 11) when specifying the number of groups according to the hierarchical clustering. The synchrony group convolution based on composite synchrony is a compromise between phase 

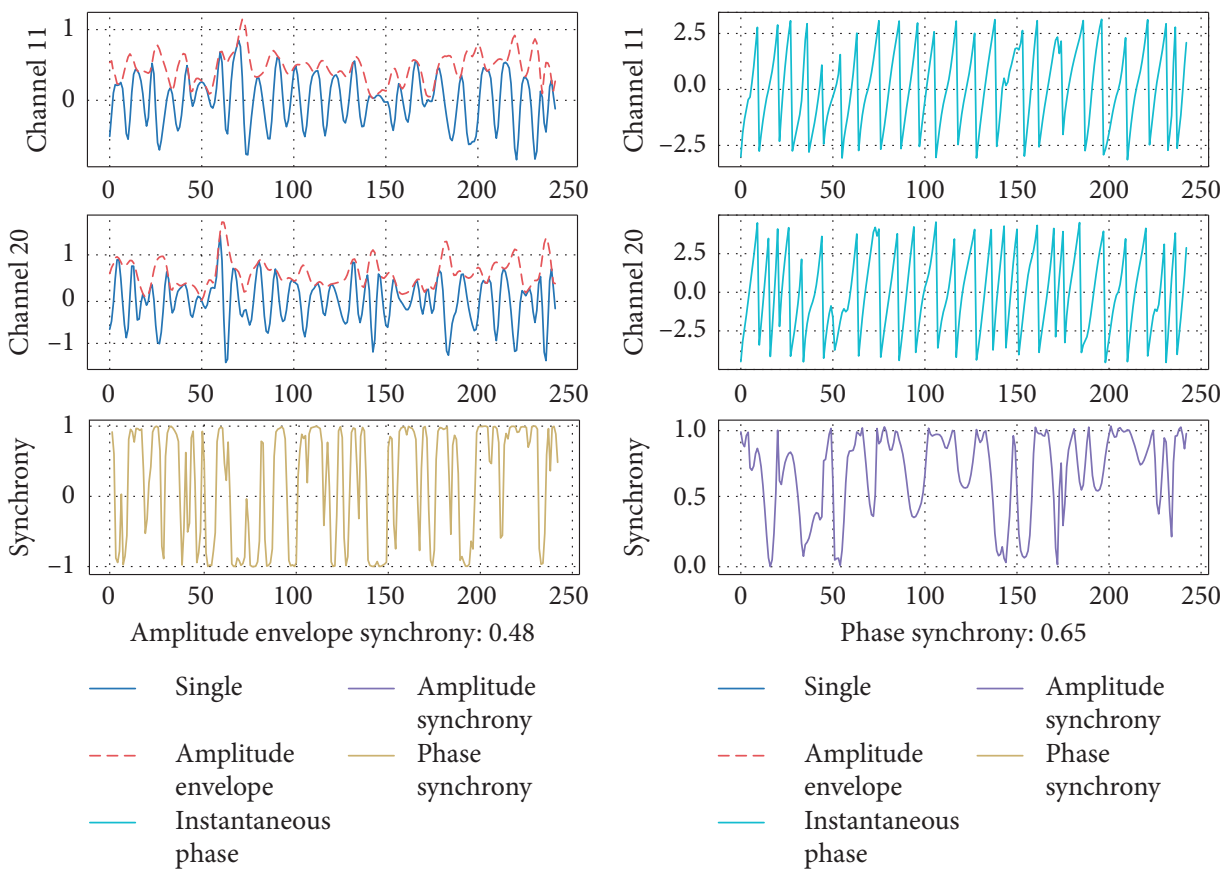

(a)
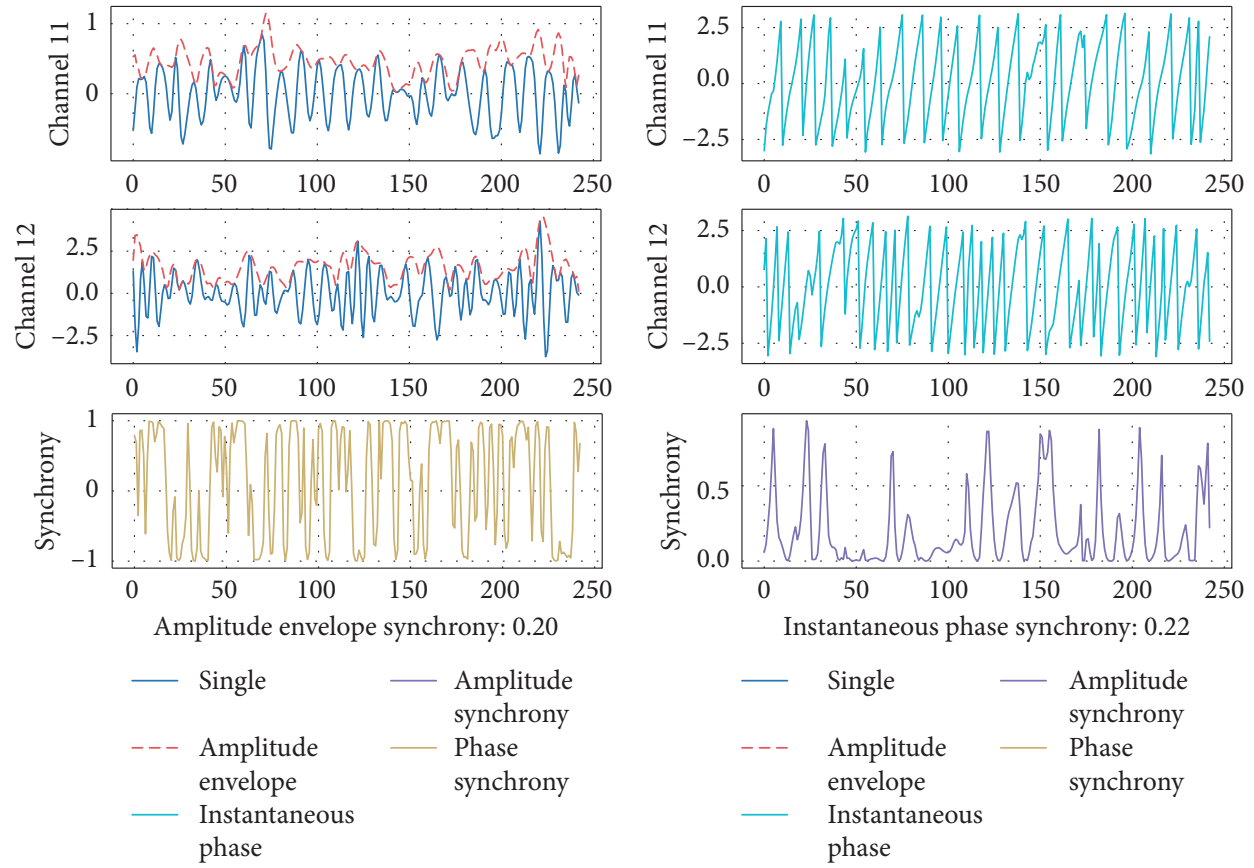

(b)

FIGURE 6: Synchrony analysis based on instantaneous phase and amplitude envelope. (a) The lateral acceleration of the bogie 1 in the middle (channel 11) and the lateral acceleration of the axle box 1 (channel 20). (b) The lateral acceleration (channel 11) and the vertical acceleration (channel 12) of the bogie 1 in the middle.

synchrony and amplitude envelope synchrony, which achieves the best performance among all experiments when the group number equals 4 .

The training curves for synchrony group convolutions are more stable in the initial few epochs and increase faster, which suggests that synchrony group convolutions are much easier to optimize. Besides, the model size of synchrony group convolutions is less than or equal to normal group convolutions when the number of groups is equal, owing to the unequal group sizes. 


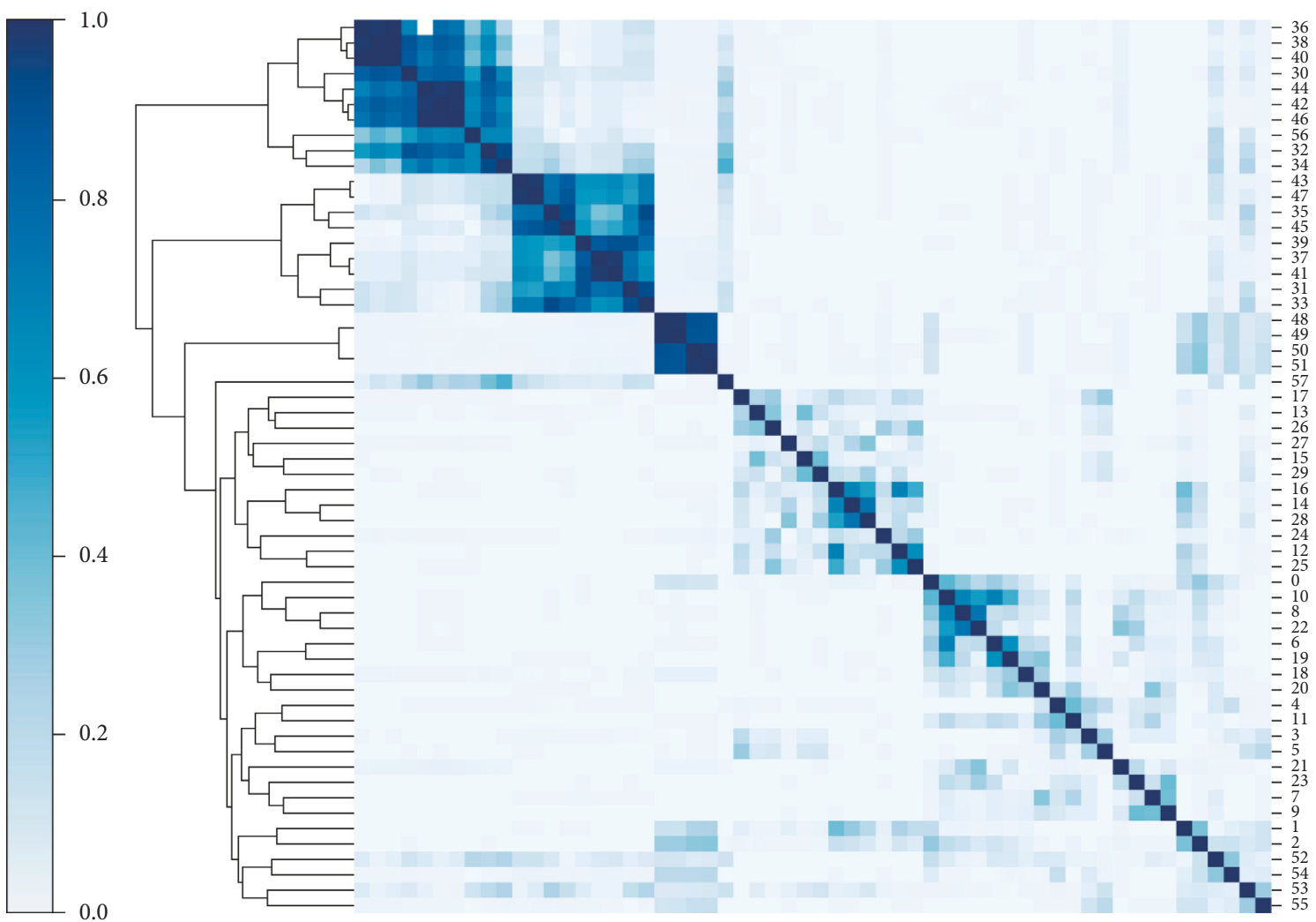

(a)
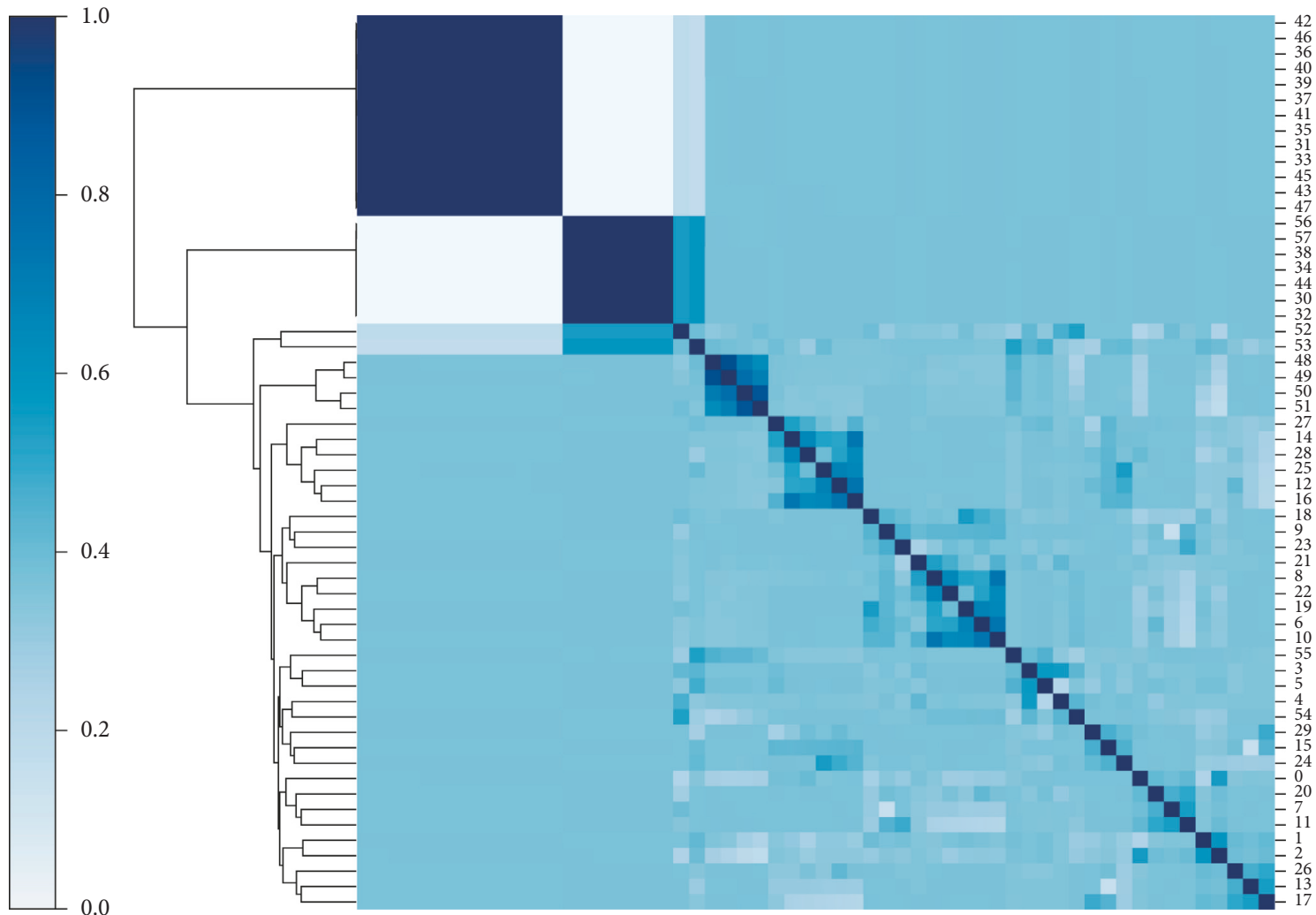

(b)

Figure 7: Continued. 


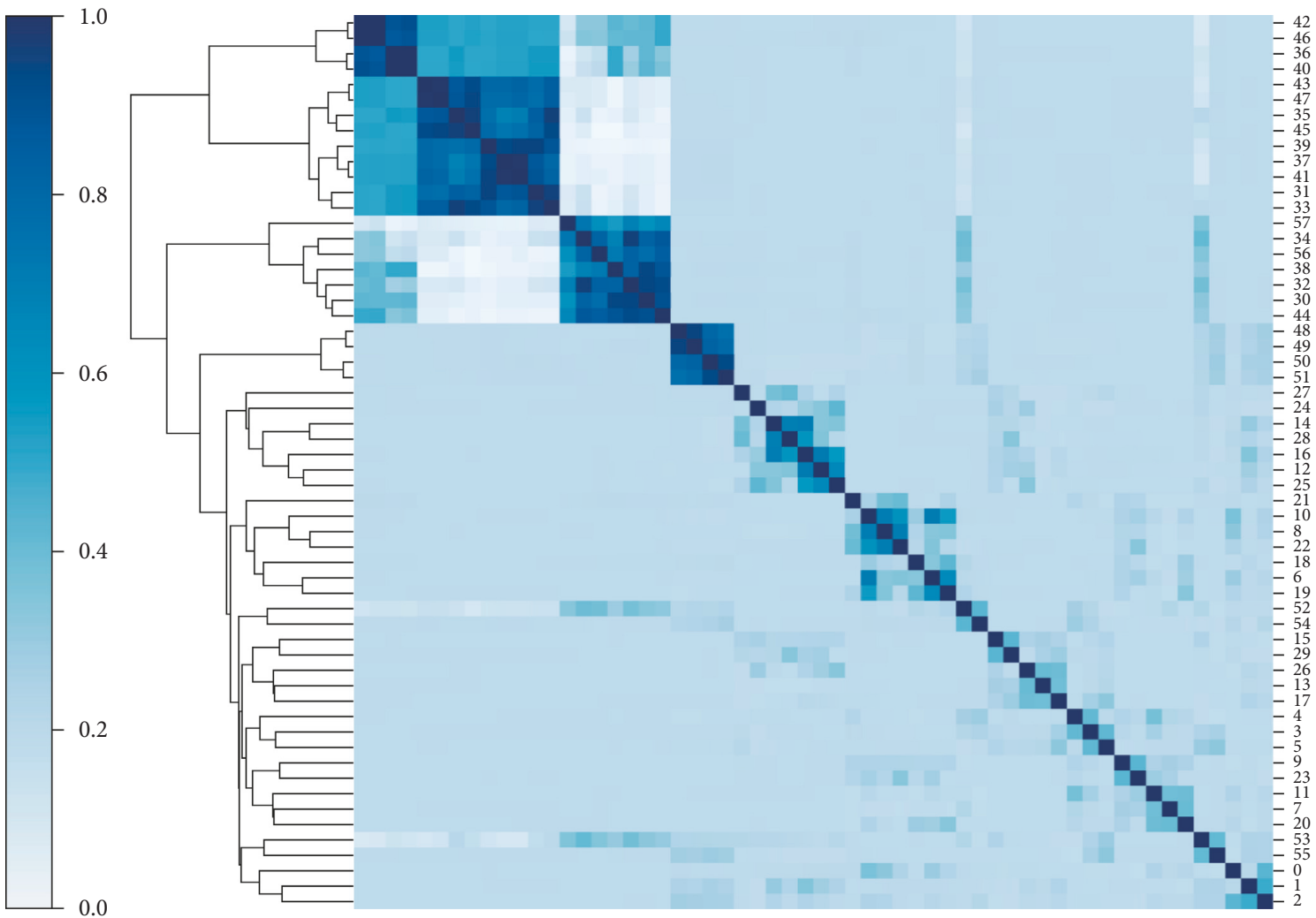

(c)

FiguRe 7: Hierarchically clustered synchrony maps for sensor channels. Hierarchical cluster map based on (a) the amplitude envelope synchrony, (b) the instantaneous phase synchrony, and (c) the composite synchrony.

TABLE 2: The experiment results of bogie fault classification.

\begin{tabular}{lcccccc}
\hline ID. & Method $^{*}$ & Model size (parameters) & Accuracy (\%) & Precision (\%) & Recall $(\%)$ & F1 score $(\%)$ \\
\hline 1 & Normal convolution & 1362391 & $73.88(\sigma=9.44)$ & $71.98(\sigma=11.98)$ & $73.88(\sigma=9.44)$ & $72.10(\sigma=11.51)$ \\
2 & GC-4 & 596501 & $87.87(\sigma=5.37)$ & $87.78(\sigma=5.58)$ & $87.87(\sigma=5.37)$ & $87.69(\sigma=5.62)$ \\
3 & GC-8 & 377290 & $69.60(\sigma=5.24)$ & $68.34(\sigma=5.83)$ & $69.60(\sigma=5.24)$ & $68.46(\sigma=5.74)$ \\
4 & GC-12 & 308632 & $65.26(\sigma=3.92)$ & $63.20(\sigma=4.58)$ & $65.26(\sigma=3.92)$ & $63.53(\sigma=4.52)$ \\
5 & ASGC-4 & 502135 & $97.79(\sigma=1.49)$ & $97.82(\sigma=1.46)$ & $97.79(\sigma=1.49)$ & $97.79(\sigma=1.49)$ \\
6 & ASGC-8 & 365719 & $91.52(\sigma=3.68)$ & $91.57(\sigma=3.68)$ & $91.52(\sigma=3.68)$ & $91.45(\sigma=3.72)$ \\
7 & ASGC-12 & $\mathbf{3 2 6 7 4 3}$ & $\mathbf{9 5 . 1 4}(\sigma=\mathbf{2 . 6 3})$ & $\mathbf{9 5 . 2 0}(\sigma=\mathbf{2 . 6 4})$ & $\mathbf{9 5 . 1 4}(\sigma=\mathbf{2 . 6 3})$ & $\mathbf{9 5 . 1 2}(\sigma=\mathbf{2 . 6 7})$ \\
8 & PSGC-4 & 613495 & $68.22(\sigma=7.97)$ & $65.56(\sigma=10.33)$ & $68.22(\sigma=7.97)$ & $65.95(\sigma=9.87)$ \\
9 & PSGC-8 & $\mathbf{3 7 3 3 7 5}$ & $\mathbf{9 3 . 4 3}(\sigma=\mathbf{5 . 1 9})$ & $\mathbf{9 3 . 5 0}(\sigma=\mathbf{5 . 1 2})$ & $\mathbf{9 3 . 4 3}(\sigma=\mathbf{5 . 1 9})$ & $\mathbf{9 3 . 4 3 (}(\sigma=\mathbf{5 . 1 8})$ \\
10 & PSGC-12 & 347623 & $91.08(\sigma=5.17)$ & $91.15(\sigma=5.24)$ & $91.08(\sigma=5.17)$ & $90.98(\sigma=5.29)$ \\
11 & CSGC-4 & $\mathbf{5 0 4 2 2 3}$ & $\mathbf{9 8 . 2 7}(\sigma=\mathbf{1 . 7 3})$ & $\mathbf{9 8 . 2 9}(\sigma=\mathbf{1 . 7 1})$ & $\mathbf{9 8 . 2 7}(\sigma=\mathbf{1 . 7 3})$ & $\mathbf{9 8 . 2 6}(\sigma=\mathbf{1 . 7 3})$ \\
12 & CSGC-8 & 364327 & $92.67(\sigma=2.67)$ & $92.74(\sigma=2.65)$ & $92.67(\sigma=2.67)$ & $92.62(\sigma=2.71)$ \\
13 & CSGC-12 & 335095 & $92.78(\sigma=3.61)$ & $92.86(\sigma=3.62)$ & $92.78(\sigma=3.61)$ & $92.73(\sigma=3.64)$ \\
\hline
\end{tabular}

*The number behind the hyphen (-) represents the group number in normal group convolution layers or synchrony group convolution layers.

\section{Conclusion and Future Work}

This paper proposes synchrony group convolutions for multichannel monitoring systems. The proposed method is able to gather signals with similar patterns and process them with specific groups of neurons, which could extract features more effectively. Signals with significant differences would be processed with different groups of neurons, instead of sharing the same neurons, which makes the model much easier to optimize. Experiment results show that the proposed model performs much better than normal convolutions and normal group convolutions on our task, which achieves an accuracy of $98.27 \%(\sigma=1.73)$ with good computational efficiency. The proposed framework is highly practical for the multisensor monitoring system and provides a reference for other engineering fields. 

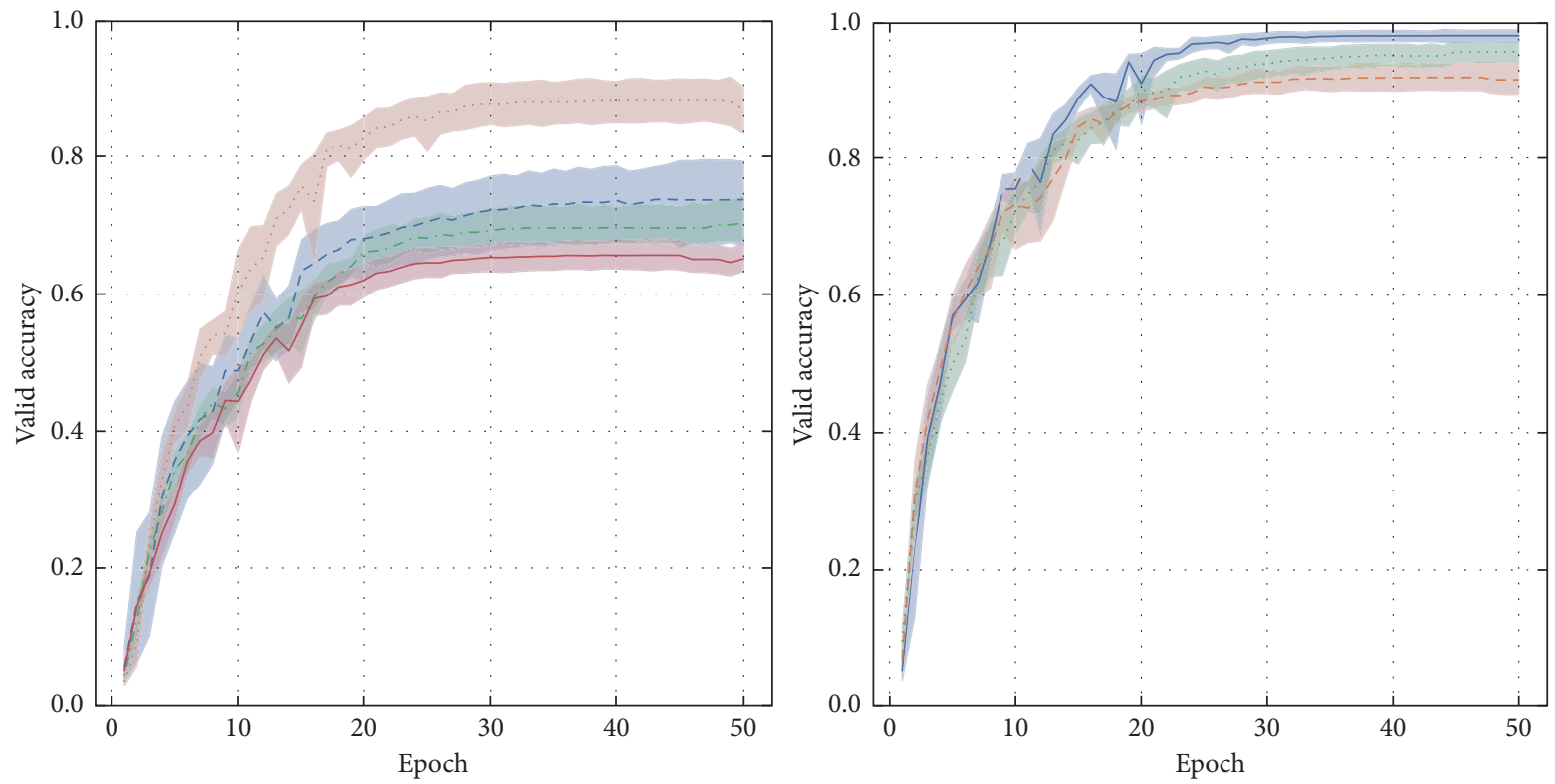

Method

Method

- - Normal convolution _... GC-8

GC-4

- GC-12

- ASGC-4

- - - ASGC-8

..... ASGC-12
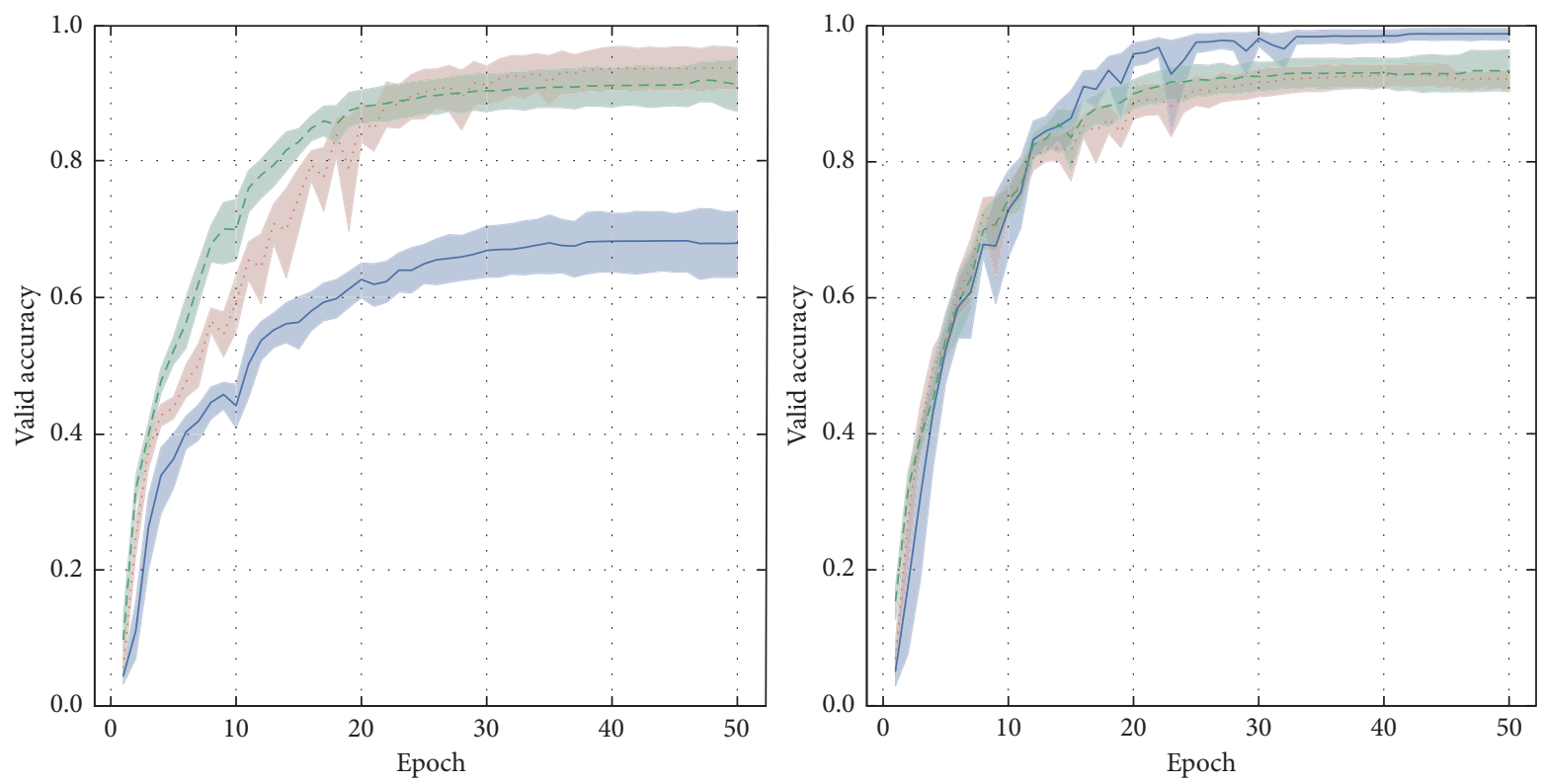

Method

Method

- PSGC-4

PSGC-8

- - - PSGC-12

- CSGC-4

CSGC-8

- - CSGC- 12

FIGURE 8: Training curves for comparative methods. Curves are averages over ten trials, and error bands give standard deviations.

As an initial work on synchrony group convolutions, the proposed approach has achieved promising performance. One possible extension of this work is the joint optimization for the number of groups and the network structure to achieve better performance. Another difficult challenge is interpretable feature cluster and fusion for multichannel fault diagnosis system, which is essential for the evaluation of reliability and uncertainty. The authors would investigate this topic in future research.

\section{Data Availability}

The data used to support the findings of this study are available from the corresponding author upon request. 


\section{Conflicts of Interest}

The authors declare that they have no conflicts of interest.

\section{References}

[1] Y. Zang, W. Shangguan, B. Cai, H. Wang, and M. G. Pecht, "Methods for fault diagnosis of high-speed railways: a review," Proceedings of the Institution of Mechanical Engineers, Part O: Journal of Risk and Reliability, vol. 233, no. 5, pp. 908-922, 2019.

[2] H. Chen and B. Jiang, "A review of fault detection and diagnosis for the traction system in high-speed trains," IEEE Transactions on Intelligent Transportation Systems, pp. 1-16, 2019.

[3] S. Y. Chong, J.-R. Lee, and H.-J. Shin, "A review of health and operation monitoring technologies for trains," Smart Structures and Systems, vol. 6, no. 9, pp. 1079-1105, 2010.

[4] R. Ström, Operational Transfer Path Analysis of Components of a High-Speed Train Bogie, Chalmers University of Technology, Gothenburg, Sweden, 2014.

[5] A. Herrero, Towards Optimization of a High Speed Train Bogie Primary Suspension, Chalmers University of Technology, Gothenburg, Sweden, 2013.

[6] E. L. Iglesias, D. J. Thompson, M. Smith, T. Kitagawa, and N. Yamazaki, "Anechoic wind tunnel tests on high-speed train bogie aerodynamic noise," International Journal of Rail Transportation, vol. 5, no. 2, pp. 87-109, 2017.

[7] Y. Lu, P. Xiang, P. Dong, X. Zhang, and J. Zeng, "Analysis of the effects of vibration modes on fatigue damage in highspeed train bogie frames," Engineering Failure Analysis, vol. 89, pp. 222-241, 2018.

[8] J. Zhu, Aerodynamic Noise of High-Speed Train Bogies, University of Southampton, Southampton, UK, 2015.

[9] Q. Wang, J. Zeng, L. Wei, and B. Zhu, "Carbody vibrations of high-speed train caused by dynamic unbalance of underframe suspended equipment," Advances in Mechanical Engineering, vol. 10, no. 12, 2018.

[10] R. Melnik and S. Koziak, "Rail vehicle suspension condition monitoring-approach and implementation," Journal of Vibroengineering, vol. 19, no. 1, pp. 487-501, 2017.

[11] O. Polach, A. Böttcher, D. Vannucci et al., "Validation of simulation models in the context of railway vehicle acceptance," Proceedings of the Institution of Mechanical Engineers, Part F: Journal of Rail and Rapid Transit, vol. 229, no. 6, pp. 729-754, 2015.

[12] D. Lebel, C. Soize, C. Funfschilling, and G. Perrin, "Highspeed train suspension health monitoring using computational dynamics and acceleration measurements," Vehicle System Dynamics, pp. 1-22, 2019.

[13] M. Jesussek and K. Ellermann, "Fault detection and isolation for a full-scale railway vehicle suspension with multiple Kalman filters," Vehicle System Dynamics, vol. 52, no. 12, pp. 1695-1715, 2014.

[14] Z. Mao, G. Tao, B. Jiang, and X.-G. Yan, "Adaptive compensation of traction system Actuator failures for high-speed trains," IEEE Transactions on Intelligent Transportation Systems, vol. 18, no. 11, pp. 2950-2963, 2017.

[15] J. Feng, J. Xu, W. Liao, and Y. Liu, "Review on the traction system sensor technology of a rail transit train," Sensors, vol. 17, no. 6, p. 1356, 2017.

[16] Y. Cheng, Z. Wang, and W. Zhang, "A novel conditionmonitoring method for axle-box bearings of high-speed trains using temperature sensor signals," IEEE Sensors Journal, vol. 19, no. 1, pp. 205-213, 2019.

[17] J. Ding, W. Zhao, B. Miao, and J. Lin, "Adaptive sparse representation based on circular-structure dictionary learning and its application in wheelset-bearing fault detection," Mechanical Systems and Signal Processing, vol. 111, pp. 399422, 2018.

[18] J. Yin and W. Zhao, "Fault diagnosis network design for vehicle on-board equipments of high-speed railway: a deep learning approach," Engineering Applications of Artificial Intelligence, vol. 56, pp. 250-259, 2016.

[19] C. Li, S. Luo, C. Cole, and M. Spiryagin, "An overview: modern techniques for railway vehicle on-board health monitoring systems," Vehicle System Dynamics, vol. 55, no. 7, pp. 1045-1070, 2017.

[20] L. Gasparetto, S. Alfi, and S. Bruni, "Data-driven conditionbased monitoring of high-speed railway bogies," International Journal of Rail Transportation, vol. 1, no. 1-2, pp. 42-56, 2013.

[21] H. Chen, B. Jiang, W. Chen, and H. Yi, "Data-driven detection and diagnosis of incipient faults in electrical drives of highspeed trains," IEEE Transactions on Industrial Electronics, vol. 66, no. 6, pp. 4716-4725, 2019.

[22] Z. Zhuang, J. Ding, A. C. Tan, Y. Shi, and J. Lin, "Fault detection of high-speed train wheelset bearing based on impulse-envelope manifold," Shock and Vibration, vol. 2017, Article ID 2104720, 17 pages, 2017.

[23] Y. LeCun, Y. Bengio, and G. Hinton, “Deep learning," Nature, vol. 521, no. 7553, pp. 436-444, 2015.

[24] J. Schmidhuber, "Deep learning in neural networks: an overview," Neural Networks, vol. 61, pp. 85-117, 2015.

[25] P. Henriquez, J. B. Alonso, M. A. Ferrer, and C. M. Travieso, "Review of automatic fault diagnosis systems using audio and vibration signals," IEEE Transactions on Systems, Man, and Cybernetics: Systems, vol. 44, no. 5, pp. 642-652, 2014.

[26] A. Bustos, H. Rubio, C. Castejón, and J. García-Prada, "EMDbased methodology for the identification of a high-speed train running in a gear operating state," Sensors, vol. 18, no. 3, p. 793, 2018.

[27] Y. Wu and W. Jin, "A fault diagnosis scheme for high-speed train bogie based on depth-wise convolution," in Proceedings of the 2018 IEEE International Conference on Progress in Informatics and Computing (PIC), pp. 169-174, Suzhou, China, December 2018.

[28] S. Kraft, J. Causse, and A. Martinez, "Black-box modelling of nonlinear railway vehicle dynamics for track geometry assessment using neural networks," Vehicle System Dynamics, vol. 57, no. 9, pp. 1241-1270, 2019.

[29] H. Hu, B. Tang, X. Gong, W. Wei, and H. Wang, "Intelligent fault diagnosis of the high-speed train with big data based on deep neural networks," IEEE Transactions on Industrial Informatics, vol. 13, no. 4, pp. 2106-2116, 2017.

[30] D. Peng, Z. Liu, H. Wang, Y. Qin, and L. Jia, "A novel deeper one-dimensional CNN with residual learning for fault diagnosis of wheelset bearings in high-speed trains," IEEE Access, vol. 7, pp. 10278-10293, 2019.

[31] Y. Wu, W. Jin, J. Ren, and Z. Sun, "A multi-perspective architecture for high-speed train fault diagnosis based on variational mode decomposition and enhanced multi-scale structure," Applied Intelligence, 2019.

[32] J. Ning, Q. Liu, H. Ouyang, C. Chen, and B. Zhang, "A multisensor fusion framework for detecting small amplitude hunting of high-speed trains," Journal of Vibration and Control, vol. 24, no. 17, pp. 3797-3808, 2018. 
[33] Y. Liu, X. Wang, J. Lin, and W. Zhao, "Correlation analysis of motor current and chatter vibration in grinding using complex continuous wavelet coherence," Measurement Science and Technology, vol. 27, no. 11, 2016.

[34] K. Hong and H. Huang, "Power Transformer Fault Diagnosis Based on Vibration Correlation Analysis," in Proceedings of the ASME 2014 International Mechanical Engineering Congress and Exposition, Montreal, Canada, November 2014.

[35] B. Kestner, T. Lieuwen, C. Hill, L. Angello, J. Barron, and C. A. Perullo, "Correlation analysis of multiple sensors for industrial gas turbine compressor blade health monitoring," Journal of Engineering for Gas Turbines and Power, vol. 137, no. 11, Article ID 112605, 2015.

[36] A. Córdova-Palomera, C. Tornador, C. Falcón et al., "Environmental factors linked to depression vulnerability are associated with altered cerebellar resting-state synchronization," Scientific Reports, vol. 6, no. 1, p. 37384, 2016.

[37] M. Pedersen, A. Omidvarnia, A. Zalesky, and G. D. Jackson, "On the relationship between instantaneous phase synchrony and correlation-based sliding windows for time-resolved fMRI connectivity analysis," NeuroImage, vol. 181, pp. 85-94, 2018.

[38] C. V. Parise and M. O. Ernst, "Correlation detection as a general mechanism for multisensory integration," Nature Communications, vol. 7, Article ID 11543, 2016.

[39] M. Feldman, "Hilbert transform in vibration analysis," Mechanical Systems and Signal Processing, vol. 25, no. 3, pp. 735-802, 2011.

[40] J. C. F. de Winter, S. D. Gosling, and J. Potter, "Comparing the Pearson and spearman correlation coefficients across distributions and sample sizes: a tutorial using simulations and empirical data," Psychological Methods, vol. 21, no. 3, pp. 273-290, 2016.

[41] M. Venouziou and H. Zhang, "Characterizing the Hilbert transform by the bedrosian theorem," Journal of Mathematical Analysis and Applications, vol. 338, no. 2, pp. 14771481, 2008.

[42] F. Murtagh and P. Contreras, "Algorithms for hierarchical clustering: an overview," Wiley Interdisciplinary Reviews: Data Mining and Knowledge Discovery, vol. 2, no. 1, pp. 86-97, 2012.

[43] O. Yim and K. T. Ramdeen, "Hierarchical cluster Analysis: comparison of three linkage measures and application to psychological data," The Quantitative Methods for Psychology, vol. 11, no. 1, pp. 8-21, 2015.

[44] A. Krizhevsky, I. Sutskever, and G. E. Hinton, "ImageNet classification with deep convolutional neural networks," in Proceedings of the 25th International Conference on Neural Information Processing Systems, vol. 1, pp. 1097-1105, Lake Tahoe, NV, USA, December 2012.

[45] Y. Ioannou, D. Robertson, R. Cipolla, and A. Criminisi, “Deep roots: improving CNN efficiency with hierarchical filter groups," in Proceedings of the IEEE Conference on Computer Vision and Pattern Recognition, pp. 1231-1240, Honolulu, HI, USA, July 2017. 


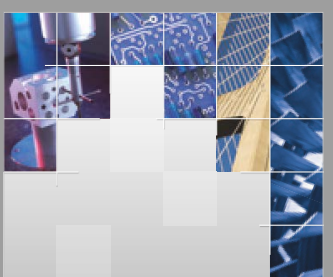

\section{Enfincering}
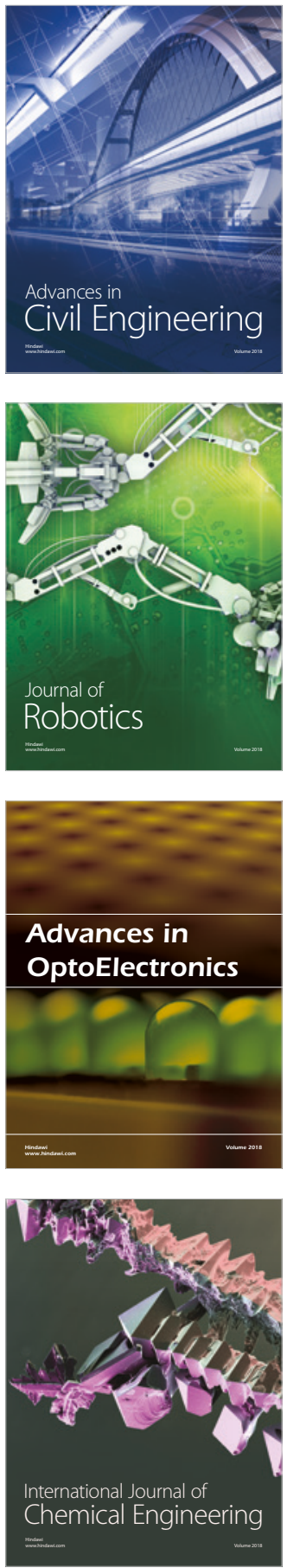

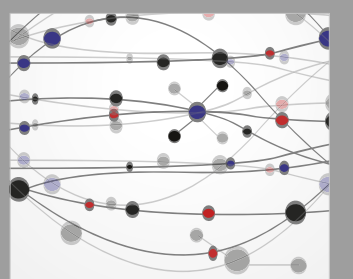

\section{Rotating \\ Machinery}

The Scientific World Journal

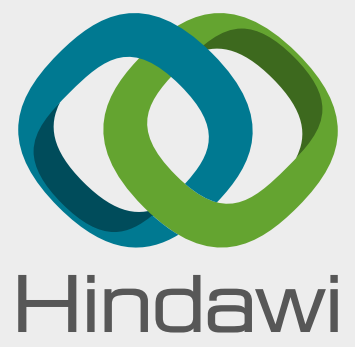

Submit your manuscripts at

www.hindawi.com
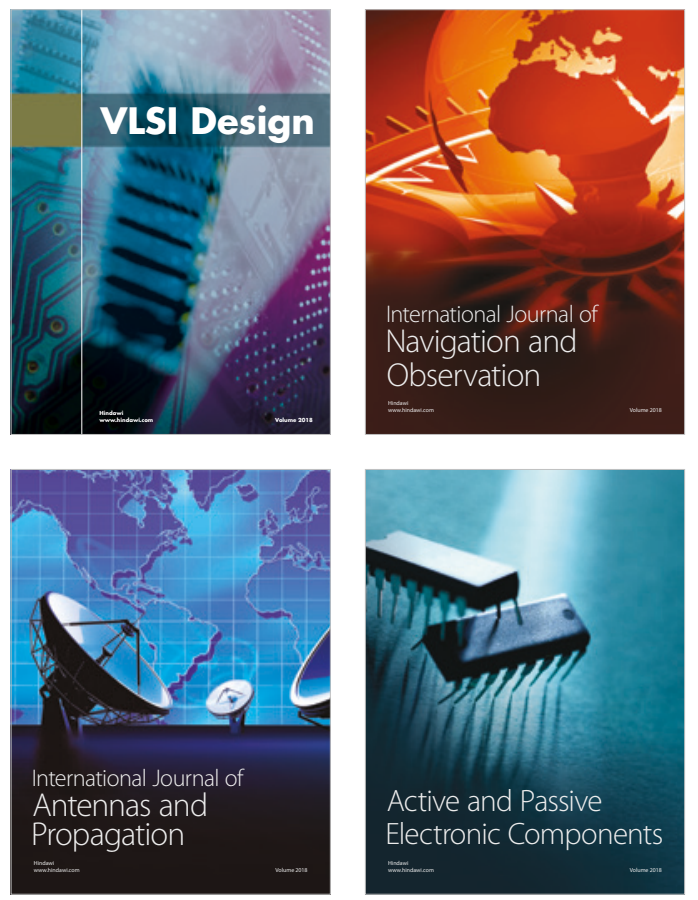
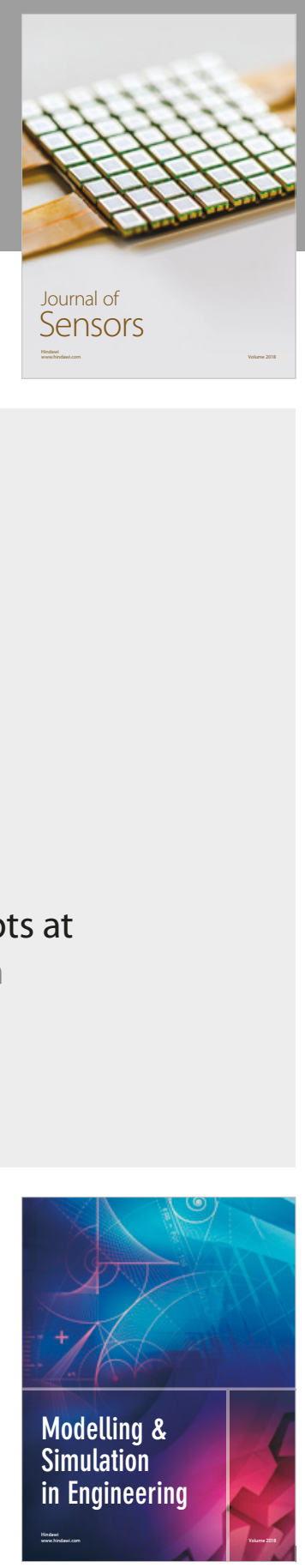

\section{Advances \\ Multimedia}
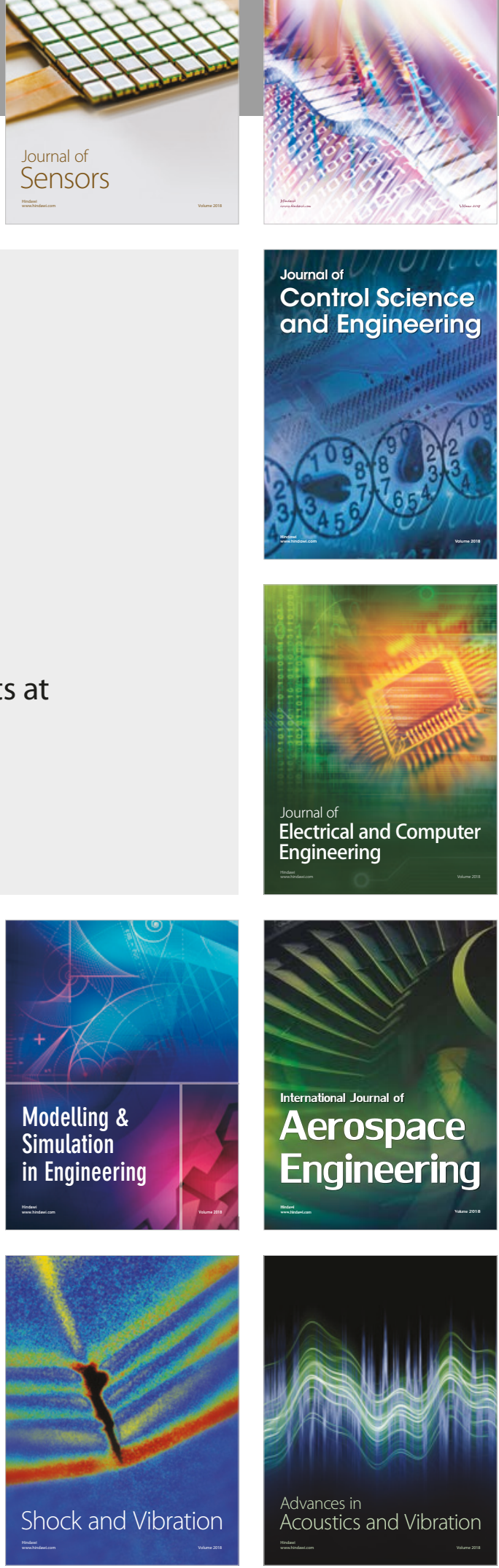